\title{
KED-AES algorithm: combined key encryption decryption and advance encryption standard algorithm
}

Edwin R. Arboleda, Carla Eunice R. Fenomeno, Joshua Z. Jimenez

Department of Computer and Electronics Engineering, College of Engineering and Information Technology, Cavite State University, Philippines

\begin{tabular}{l} 
Article Info \\
\hline Article history: \\
Received Nov 21, 2018 \\
Revised Jan 16, 2019 \\
Accepted Feb 1, 2019 \\
\hline Keywords: \\
Advance encryption standard \\
Cryptography \\
Key encryption decryption
\end{tabular}

Key encryption decryption

\begin{abstract}
Two existing cryptosystems are being combined and proposed. It is the enhanced combination of KED (Key Encryption Decryption), a cryptosystem that uses modulo 69 and the AES (Advance Encryption Standard) cryptography. The strength of the KED is that the keys are being used by the sender and the receiving end. The AES is easy to implement and has good defense against various attacks such as hacking.
\end{abstract}

Copyright (C) 2019 Institute of Advanced Engineering and Science. All rights reserved.

\section{Corresponding Author:}

Edwin R. Arboleda,

Department of Computer and Electronics Engineering,

College of Engineering and Information Technology, Cavite State University,

Indang, Cavite, Philippines.

Email: edwin.r.arboleda@cvsu.edu.ph

\section{INTRODUCTION}

Cryptography is the science of encrypting and decrypting information. It is the art of securing data or information by transforming it into another unreadable format wherein a message is hidden from anyone who will read it and only the intended receiver will be able to convert it and reveal the hidden message. The main goal of cryptography is to secure the data from illegal access $[1,2]$.

Based on [3,4], it stated that Cryptography is the scientific establishment on which one forms secure frameworks. It contemplates methods for safely putting away, transmitting, and handling data. Understanding what cryptographic primitives can do, and how they can be made together, is important to construct secure frameworks, however not enough. A few extra contemplations go into the outline of secure frameworks, and they are secured in different Berkeley graduate courses on security [2].

Cryptography is about correspondence within the sight of an enemy. It includes numerous issues (encryption, verification, key appropriation to give some examples). The field of current cryptography gives a hypothetical establishment considering which we may comprehend what precisely these issues are, the manner by which to assess conventions that indicate to explain them, and how to construct conventions in whose security we can have. We present the essential issues by talking about the issue of encryption [5].

According to [6], Cryptography is the place security designing meets arithmetic. It gives us the apparatuses that underlie most advanced security conventions. It is presumably the key empowering innovation for securing appropriated frameworks, yet it is shockingly difficult to do right. "Conventions," cryptography has frequently been utilized to secure the wrong things or used to ensure them in the wrong way. We'll see bounty more cases when we begin looking in detail at genuine applications [7]. 
Cryptography is the exploration of writing in mystery code and is an antiquated craftsmanship; the initially reported utilization of cryptography in composing goes back to around 1900 B.C. at the point when an Egyptian copyist utilized non-standard symbolic representations as a part of an engraving [8]. A few specialists contend that cryptography showed up suddenly at some point in the wake of composing was developed, with applications running from strategic letters to war-time fight arranges. It is nothing unexpected, then, that new types of cryptography came not long after the broad improvement of PC correspondences [9]. In information and broadcast communications, cryptography is fundamental when imparting over any untrusted medium, which incorporates pretty much any system, especially the internet [8].

There are two basic types of encryption, symmetric algorithm and asymmetric algorithm. According to [10] symmetric algorithms also called "secret key" uses the same key for both encryption and decryption and asymmetric algorithms or "public key" uses different keys for encryption and decryption [11]. A symmetric cryptosystem (or private key cryptosystem) utilizes one and only key for both encryption and unscrambling of the information. The key utilized for encryption and unscrambling is called the private key and just individuals who are approved for the encryption/unscrambling would know it. In a symmetric cryptosystem, the encoded message is sent over without any open keys joined to it while asymmetric or the public key there are two distinctive keys utilized for the encryption and decoding of information [8]. The key utilized for encryption is kept open thus as called open key, and the unscrambling key is kept mystery and called private key. The keys are created in a manner that it is difficult to get the private key from people in general key. The transmitter and the beneficiary both have two keys in an Asymmetric framework. Be that as it may, the private key is kept private and not sent over with the message to the beneficiary, in spite of the fact that the general population key is this is further explained by [11].

The advantages and disadvantages of symmetric and asymmetric cryptosystem was stated by [12], A symmetric cryptosystem is speedier, encoded information be exchanged on the connection regardless of the possibility that there is a probability that the information will be caught. Since there is no key transmitted with the information, the odds of information being decoded are invalid, it utilizes secret word validation to demonstrate the beneficiary's character and a framework just which has key can unscramble a message are the benefits of symmetric cryptosystem while in Asymmetric cryptosystem [13], cryptography there is no requirement for trading keys, along these lines wiping out the key conveyance issue [14], the essential preferred standpoint of open key cryptography is expanded security: the private keys absolutely never should be transmitted or uncovered to anybody, and in conclusion it can give advanced marks that can be denied [15]. The disadvantages of symmetric cryptosystem have an issue of key transportation. The private key is to be transmitted to the getting framework before the real message is to be transmitted [16, 17]. Each method for electronic correspondence channels. In this way, the main secure method for trading keys would trade them by and by and can't give advanced mark that can't be revoked. While in asymmetric cryptosystem an impediment of utilizing open key cryptography for encryption is speed: there are well known mystery key encryption techniques which are altogether speedier than any right now accessible open key encryption strategy [18].

This standard indicates the Rijndael calculation [19, 20], a symmetric square figure that can prepare information squares of 128 bits, utilizing figure keys with lengths of 128, 192, and 256 bits [21]. Rijndael was intended to handle extra piece sizes and key lengths, nonetheless they are not embraced in this standard. All through the rest of this standard, the calculation determined thus will be alluded to as "the AES algorithm." The algorithm might be utilized with the three different key lengths, mention above, and in this way, these different "flavors" might be alluded to as "AES-128", "AES-192", and"AES-256 [4]".

Warjir [22] proposed a new symmetric key algorithm called as KED (Key Encryption Decryption) and a new key generation method. Using modulo 69 and inverse modulo69, the proposed algorithm was used for encryption and decryption process, in which the same key is used both for encryption and decryption.

\section{RESEARCH METHOD}

The authors proposed a hybrid of KED that uses modulo 69, CHAOS-based cryptosystem, and AES algorithm. The key generation used in this hybrid uses that of KED and all the algorithm are used in encryption and decryption process [18]. The s-box and inverse s-box tables will be used in encryption and decryption respectively; they will be part of the process itself and not in the sent message. The Encryption process is shown in Figure 1 and The Decryption process is shown in Figure 2. 


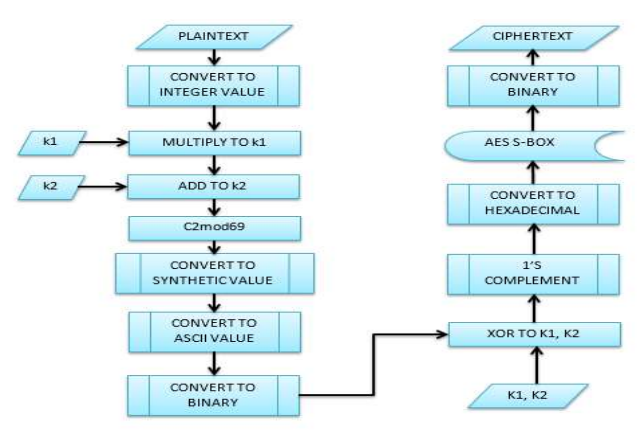

Figure 1. Encryption process

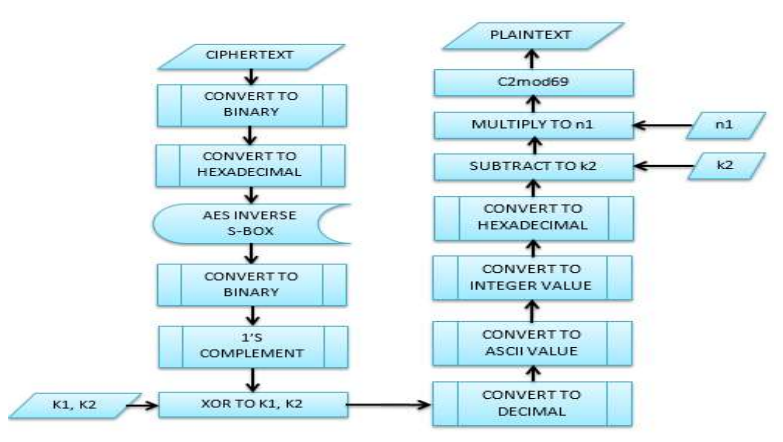

Figure 2. Decryption process [23]

\section{Key generation algorithm}

KED Key Generation:

a. Generating ' $\mathrm{k} 2$ ', firstly user enters a key. The length of the key is stored in ' $\mathrm{kl}$ '. Hence $\mathrm{k} 2$ is generated as follows:

$\mathrm{K} 2=\left(\sum_{i=0}^{k l-1} 2^{i} * \mathrm{kl} * \mathrm{val}\right) \bmod \mathrm{m}$

b. Where, ' $\mathrm{i}$ ' is the position of each character in the key. ' $\mathrm{kl}$ ' is the length of the key. 'val' is the integer value that has been assign to each character as shown in Figure 3.

c. For ' $\mathrm{k} 1$ ', select any natural number say ' $\mathrm{k} 1$ ' where $\mathrm{k} 1 \neq 0$ and must be relatively prime to ' $\mathrm{m}$ ' (i.e., ' $\mathrm{k} 1$ ' should not have factors in common with ' $\mathrm{m}$ ').

d. Find inverse modulo69 of ' $\mathrm{k} 1$ ' and store it in ' $\mathrm{n} 1$ '

Chaos Key Generation:

a. Convert $\mathrm{k} 1$ and $\mathrm{k} 2$ to its 8-bit binary equivalent.

b. Get the gray code of the binary form of $\mathrm{k} 1$ and $\mathrm{k} 2$, denote as $\mathrm{J} 1$ and $\mathrm{J} 2$.

\section{Encryption}

KED:

\begin{tabular}{|c|c|c|c|c|c|c|c|c|}
\hline $\mathbf{A}$ & B & C & D & $\mathbf{E}$ & $\mathbf{F}$ & $G$ & $\mathbf{H}$ & $\mathbf{I}$ \\
\hline 1 & 2 & 3 & 4 & 5 & 6 & 7 & 8 & 9 \\
\hline $\mathbf{J}$ & $\mathrm{K}$ & $\mathbf{L}$ & MF & $N$ & 0 & $\mathbf{P}$ & Q & $\mathbf{R}$ \\
\hline 10 & 11 & 12 & 13 & 14 & 15 & 16 & 17 & 18 \\
\hline$S$ & $\mathbf{T}$ & $\mathbf{U}$ & $\mathbf{v}$ & $\mathbf{w}$ & $x$ & $\mathbf{Y}$ & $\mathbf{z}$ & 0 \\
\hline 19 & 20 & 21 & 22 & 23 & 24 & 25 & 26 & 27 \\
\hline 1 & 2 & 3 & 4 & 5 & 6 & 7 & 8 & 9 \\
\hline 28 & 29 & 30 & 31 & 32 & 33 & 34 & 35 & 36 \\
\hline space & $!$ & " & $\#$ & $s$ & $\%$ & $\&$ & - & C \\
\hline 37 & 38 & 39 & 40 & 41 & 42 & 43 & 44 & 45 \\
\hline ) & * & + & , & - & $\cdot$ & $f$ & : & $;$ \\
\hline 46 & 47 & 48 & 49 & 50 & 51 & 52 & 53 & 54 \\
\hline$<$ & $=$ & $>$ & $?$ & (a) & [ & 1 & 1 & $\wedge$ \\
\hline 55 & 56 & 57 & 58 & 59 & 60 & 61 & 62 & 63 \\
\hline- & & \{ & 1 & 3 & $\sim$ & & & \\
\hline 64 & 65 & 66 & 67 & 68 & 69 & & & \\
\hline
\end{tabular}

Figure 3. Synthetic values of alphabets [23]

Int. J. of Adv. in Appl. Sci. Vol. 8, No. 1, March 2019: 44 - 53 
a. Firstly, substitute or assign integer value for plain text.

b. Multiply Synthetic value with first key i.e., k1.

c. Now add the result from step 2 above with second key i.e., k2.

d. Then calculate with modulo69.

CHAOS:

a. Convert mi to 8-bit binary equivalent.

b. XOR the 8-bit binary with the corresponding $\mathrm{k}$.

c. Get the 1's complement, denoted as w.

AES:

a. Convert the binary to hexadecimal

b. Apply the S-box to each value as shown in Figure 4, denoted as W1.

c. The first digit will be the row $\mathrm{x}$, and second digit will be the column $\mathrm{y}$.

d. Convert to binary the row $\mathrm{x}$ and column $\mathrm{y}$.

e. Convert to decimal the 8-bit binary for the cipher text.

\begin{tabular}{|c|c|c|c|c|c|c|c|c|c|c|c|c|c|c|c|c|c|}
\hline & \multicolumn{16}{|c|}{$y$} \\
\hline & & 0 & 1 & 2 & 3 & 4 & 5 & 6 & 7 & 8 & 9 & a & b & c & d & e & $\mathbf{f}$ \\
\hline \multirow{16}{*}{$x$} & 0 & 63 & $7 \mathrm{c}$ & 77 & $7 b$ & 12 & $6 b$ & $6 f$ & c5 & 30 & 01 & 67 & $2 b$ & $\mathrm{fe}$ & d7 & $a b$ & 76 \\
\hline & 1 & ca & 82 & c9 & $7 d$ & $\mathrm{fa}$ & 59 & 47 & fo & ad & $\mathrm{d} 4$ & a2 & af & $9 \mathrm{c}$ & a 4 & 72 & со \\
\hline & 2 & b7 & $\mathrm{fd}$ & 93 & 26 & 36 & $3 f$ & 87 & $\mathrm{ce}$ & 34 & a5 & e5 & $\mathrm{fl}$ & 71 & d8 & 31 & 15 \\
\hline & 3 & 04 & c7 & 23 & $c 3$ & 18 & 96 & 05 & $9 \mathrm{a}$ & 07 & 12 & 80 & $e 2$ & $\overline{c b}$ & 27 & b2 & 75 \\
\hline & 4 & 09 & 83 & $2 \mathrm{e}$ & Ia & $1 \mathrm{bb}$ & $6 e$ & $5 a$ & aO & 52 & $3 b$ & d6 & b3 & 29 & e3 & $2 f$ & 84 \\
\hline & 5 & 53 & d1 & 00 & ed & 20 & fe & b1 & $5 b$ & $6 a$ & $c b$ & be & 39 & $4 a$ & $4 c$ & 58 & cf \\
\hline & 6 & do & ef & aa & $\mathrm{fb}$ & 43 & $4 d$ & 33 & 85 & 45 & 19 & 02 & $7 f$ & So & $3 c$ & 96 & as \\
\hline & 7 & 51 & $a 3$ & 40 & $8 f$ & 92 & $9 \mathrm{~d}$ & 38 & IS & $b c$ & b6 & $\mathrm{da}$ & 21 & 10 & $\pi$ & $\sqrt{3}$ & d2 \\
\hline & 8 & ed & $\alpha c$ & 13 & $\infty$ & $5 f$ & 97 & 44 & 17 & c4 & a7 & $7 \mathrm{e}$ & $3 \mathrm{~d}$ & 64 & 5d & 19 & 73 \\
\hline & 9 & 60 & 81 & $4 f$ & de & 22 & $2 a$ & 90 & 88 & 46 & ee & b8 & 14 & de & $5 e$ & $O b$ & $\mathrm{db}$ \\
\hline & $\mathbf{a}$ & eo & 32 & $3 a$ & $\mathrm{Oa}$ & 49 & 06 & 24 & $5 c$ & $c 2$ & d3 & $\mathrm{ac}$ & 62 & 91 & 95 & e4 & 79 \\
\hline & b & e7 & c8 & 37 & $6 d$ & $8 d$ & d5 & $4 \mathrm{e}$ & a9 & $6 c$ & 56 & 14 & ea & 65 & $7 a$ & ae & 08 \\
\hline & c & ba & 78 & 25 & $2 \mathrm{e}$ & $1 \mathrm{c}$ & a6 & b4 & c6 & e8 & dd & 74 & If & $4 b$ & $\mathrm{db}$ & $8 b$ & $8 a$ \\
\hline & d & 70 & $3 e$ & b5 & 66 & 48 & 03 & 16 & De & 61 & 35 & 57 & b9 & 86 & cl & Id & $9 e$ \\
\hline & e & el & 18 & 98 & 11 & 69 & d9 & $8 \mathrm{e}$ & 94 & $9 b$ & le & 87 & e9 & $\boldsymbol{c e}$ & 55 & 28 & df \\
\hline & $\mathrm{f}$ & $8 \mathrm{c}$ & al & 89 & Odd & $b f$ & e6 & 42 & 68 & 41 & 99 & $2 \mathrm{~d}$ & of & bo & 54 & $b b$ & 16 \\
\hline
\end{tabular}

Figure 4. AES S-Box [23]

\section{Decryption}

AES:

a. Convert to binary to hexadecimal.

b. Apply the inverse S-box as shown in Figure 5, denoted as W2.

c. Covert to binary.

\begin{tabular}{|c|c|c|c|c|c|c|c|c|c|c|c|c|c|c|c|c|c|}
\hline & \multicolumn{16}{|c|}{$y$} \\
\hline & & 0 & $T$ & 2 & 3 & 4 & 5 & 6 & 7 & 8 & 9 & $a$ & b & $\bar{c}$ & $d$ & $\bar{e}$ & $\mathrm{f}$ \\
\hline & 0 & 52 & 09 & $6 a$ & d5 & 30 & 36 & 25 & 38 & bf & 40 & $a 3$ & $9 e$ & 81 & $\mathrm{f3}$ & d7 & $\mathrm{fb}$ \\
\hline & 1 & $7 \mathrm{c}$ & $\mathrm{c} 3$ & 39 & 82 & $9 b$ & $2 f$ & $\mathrm{ff}$ & 87 & 34 & $8 e$ & 43 & 44 & $\mathrm{c4}$ & de & c9 & $\mathrm{cb}$ \\
\hline & 2 & 54 & $7 b$ & 94 & 32 & a6 & $c 2$ & 23 & $3 \mathrm{~d}$ & $\mathrm{ee}$ & $4 c$ & 95 & $0 b$ & 42 & $f_{a}$ & c3 & $4 c$ \\
\hline & 3 & 08 & $2 c$ & al & 66 & 28 & d9 & 24 & b2 & 76 & $5 b$ & $a 2$ & 49 & $6 d$ & $8 b$ & dl & 25 \\
\hline & 4 & 72 & 18 & 16 & 64 & 86 & 68 & 98 & 16 & $\mathrm{~d} 4$ & 24 & $5 e$ & $c c$ & $5 d$ & 65 & $b 6$ & 92 \\
\hline & 5 & $6 c$ & 70 & 48 & 50 & fd & ed & b9 & da & $5 \mathrm{c}$ & 15 & 46 & 57 & a7 & $8 d$ & $9 b$ & 84 \\
\hline & 6 & 90 & $d 8$ & $a b$ & 00 & $8 c$ & $b c$ & $\mathrm{~d} 3$ & $0 a$ & 17 & 04 & 58 & 05 & b8 & b3 & 45 & 06 \\
\hline & 7 & $\mathrm{~d} 0$ & $2 c$ & le & $8 f$ & $\mathrm{ca}$ & $3 f$ & of & 02 & cl & af & bd & 03 & 01 & 13 & $8 a$ & $6 b$ \\
\hline & 8 & $3 a$ & 91 & II & 41 & $4 f$ & 67 & $\mathrm{dc}$ & ca & 97 & 几 & of & $\boldsymbol{c e}$ & fo & b4 & c6 & 73 \\
\hline & 9 & 96 & $a c$ & 74 & 22 & c7 & ad & 35 & 85 & $\mathrm{c2}$ & f9 & 37 & e8 & Ic & 75 & df & $6 c$ \\
\hline & $\mathrm{a}$ & 47 & $\mathrm{fl}$ & Ia & 71 & Id & 29 & c5 & 89 & $6 f$ & b7 & 62 & $D e$ & aa & 18 & $b c$ & $\mathrm{Ib}$ \\
\hline & b & fc & 56 & $3 e$ & $4 b$ & c6 & d2 & 79 & 20 & $9 \mathrm{a}$ & $\mathrm{db}$ & co & fe & 78 & $\mathrm{~cd}$ & $5 a$ & 64 \\
\hline & $\mathrm{c}$ & If & dd & $\mathrm{a} 8$ & 33 & 88 & 07 & c7 & 31 & b1 & 12 & 10 & 59 & 27 & 80 & $\mathrm{ec}$ & $5 f$ \\
\hline & d & 60 & 51 & $7 f$ & a9 & 19 & b5 & $4 a$ & Od & $2 d$ & c5 & $7 \mathrm{a}$ & 91 & 93 & c9 & $9 \mathrm{c}$ & ef \\
\hline & $\mathrm{c}$ & 20 & $\mathrm{co}$ & $3 b$ & $4 \mathrm{~d}$ & $a c$ & $2 a$ & f5 & 60 & $\mathrm{c8}$ & $c b$ & $\mathrm{bb}$ & $3 \mathrm{c}$ & 83 & 53 & 99 & 61 \\
\hline & $f$ & 17 & $2 b$ & 04 & $7 \mathrm{e}$ & ba & 77 & d6 & 26 & el & 69 & 14 & 63 & 55 & 21 & $0 \mathrm{c}$ & $7 \mathrm{~d}$ \\
\hline
\end{tabular}

Figure 5. AES Inverse S-Box [23] 
CHAOS:

a. Get the 1's complement, denoted as ' $\mathrm{S}$ '.

b. XOR the 8-bit binary with the corresponding k.

c. Convert the 8-bit binary to its decimal equivalent, denoted as mi.

KED:

a. Assign integer value for cipher text as given in Figure 3.

b. Subtract ' $\mathrm{k} 2$ ' from above integer value.

c. Multiply above result with inverse modulo69 of ' $k 1$ ' i.e., ' $n 1$ '.

d. Finally calculate with modulo69.

\section{RESULTS AND ANALYSIS}

Message: COZY LOOBY GIVES SHARP SQUID WHO ASK FOR JOB PEN. The Encryption and Decryption are shown in Table 1-6.

Encryption:

Table 1. KED encryption

\begin{tabular}{|c|c|c|c|c|c|}
\hline $\begin{array}{l}\text { Plaintext } \\
\text { (PT) }\end{array}$ & $\begin{array}{c}\text { Integer } \\
\text { Value (V1) }\end{array}$ & $\begin{array}{c}\mathrm{V} 1 * \mathrm{~K} 1 \\
(\mathrm{C} 1)\end{array}$ & $\begin{array}{c}\mathrm{C} 1+\mathrm{K} 2 \\
(\mathrm{C} 2)\end{array}$ & $\begin{array}{c}\mathrm{C} 2 \\
\bmod 69\end{array}$ & $\begin{array}{c}\text { Synthetic } \\
\text { Value }\end{array}$ \\
\hline $\mathrm{C}$ & 3 & 15 & 21 & 21 & $\mathrm{U}$ \\
\hline $\mathrm{O}$ & 15 & 75 & 81 & 12 & $\mathrm{~L}$ \\
\hline Z & 26 & 130 & 136 & 67 & | \\
\hline $\mathrm{Y}$ & 25 & 125 & 131 & 62 & ] \\
\hline SPACE & 37 & 185 & 191 & 53 & : \\
\hline $\mathrm{L}$ & 12 & 60 & 66 & 66 & \{ \\
\hline $\mathrm{O}$ & 15 & 75 & 81 & 12 & $\mathrm{~L}$ \\
\hline $\mathrm{O}$ & 15 & 75 & 81 & 12 & $\mathrm{~L}$ \\
\hline B & 2 & 10 & 16 & 16 & $\mathrm{P}$ \\
\hline Y & 25 & 125 & 131 & 62 & ] \\
\hline SPACE & 37 & 185 & 191 & 53 & : \\
\hline $\mathrm{G}$ & 7 & 35 & 41 & 41 & $\$$ \\
\hline I & 9 & 45 & 51 & 51 & \\
\hline V & 22 & 110 & 116 & 47 & $*$ \\
\hline E & 5 & 25 & 31 & 31 & 4 \\
\hline $\mathrm{S}$ & 19 & 95 & 101 & 32 & 5 \\
\hline SPACE & 37 & 185 & 191 & 53 & : \\
\hline $\mathrm{S}$ & 19 & 95 & 101 & 32 & 5 \\
\hline $\mathrm{H}$ & 8 & 40 & 46 & 46 & ) \\
\hline A & 1 & 5 & 11 & 11 & $\mathrm{~K}$ \\
\hline $\mathrm{R}$ & 18 & 90 & 96 & 27 & 0 \\
\hline $\mathrm{P}$ & 16 & 80 & 86 & 17 & Q \\
\hline SPACE & 37 & 185 & 191 & 53 & : \\
\hline $\mathrm{S}$ & 19 & 95 & 101 & 32 & 5 \\
\hline Q & 17 & 85 & 91 & 22 & V \\
\hline U & 21 & 105 & 111 & 42 & $\%$ \\
\hline I & 9 & 45 & 51 & 51 & \\
\hline D & 4 & 20 & 26 & 26 & Z \\
\hline SPACE & 37 & 185 & 191 & 53 & : \\
\hline W & 23 & 115 & 121 & 52 & i \\
\hline $\mathrm{H}$ & 8 & 40 & 46 & 46 & ( \\
\hline 0 & 15 & 75 & 81 & 12 & U \\
\hline SPACE & 37 & 185 & 191 & 53 & : \\
\hline A & 1 & 5 & 11 & 11 & K \\
\hline $\mathrm{S}$ & 19 & 95 & 101 & 32 & 5 \\
\hline $\mathrm{K}$ & 11 & 55 & 61 & 61 & 1 \\
\hline SPACE & 37 & 185 & 191 & 53 & : \\
\hline $\mathrm{F}$ & 6 & 30 & 36 & 36 & 9 \\
\hline $\mathrm{O}$ & 15 & 75 & 81 & 12 & U \\
\hline $\mathrm{R}$ & 18 & 90 & 96 & 27 & 0 \\
\hline SPACE & 37 & 185 & 191 & 53 & : \\
\hline $\mathrm{J}$ & 10 & 50 & 56 & 56 & $=$ \\
\hline $\mathrm{O}$ & 15 & 75 & 81 & 12 & $\mathrm{U}$ \\
\hline B & 2 & 10 & 16 & 16 & $\mathrm{P}$ \\
\hline SPACE & 37 & 185 & 191 & 53 & : \\
\hline $\mathrm{P}$ & 16 & 80 & 86 & 17 & Q \\
\hline E & 5 & 25 & 31 & 31 & 4 \\
\hline $\mathrm{N}$ & 14 & 70 & 76 & 7 & G \\
\hline & 51 & 225 & 261 & 54 & 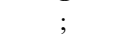 \\
\hline
\end{tabular}

Int. J. of Adv. in Appl. Sci. Vol. 8, No. 1, March 2019: 44 - 53 
Int. J. of Adv. in Appl. Sci.

ISSN: $2252-8814$

Table 2. CHAOS encryption

\begin{tabular}{|c|c|c|c|c|}
\hline ASCII Value & Binary & Gray coded (K1 \& K2) & XOR & 1's Complement \\
\hline 85 & 01010101 & 00000111 & 01010010 & 10101101 \\
\hline 76 & 01001100 & 00000101 & 01001001 & 10110110 \\
\hline 124 & 01111100 & 00000111 & 01111011 & 10000100 \\
\hline 93 & 01011101 & 00000101 & 01011000 & 10100111 \\
\hline 58 & 00111010 & 00000111 & 00111101 & 11000010 \\
\hline 123 & 01111010 & 00000101 & 01111110 & 10000001 \\
\hline 76 & 01001100 & 00000111 & 01001011 & 10110100 \\
\hline 76 & 01001100 & 00000101 & 01001001 & 10110110 \\
\hline 80 & 01010000 & 00000111 & 01010111 & 10101000 \\
\hline 93 & 01011101 & 00000101 & 01011000 & 10100111 \\
\hline 58 & 00111010 & 00000111 & 00111101 & 11000010 \\
\hline 36 & 00100100 & 00000101 & 00100001 & 11011110 \\
\hline 46 & 00100100 & 00000111 & 00100011 & 11011100 \\
\hline 42 & 00101010 & 00000101 & 00101111 & 11010000 \\
\hline 52 & 00110100 & 00000111 & 00110011 & 11001100 \\
\hline 53 & 00110101 & 00000101 & 00110000 & 11001111 \\
\hline 58 & 00111010 & 00000111 & 00111101 & 11000010 \\
\hline 53 & 00110101 & 00000101 & 00110000 & 11001111 \\
\hline 41 & 00101001 & 00000111 & 00101110 & 11010001 \\
\hline 75 & 01001011 & 00000101 & 01001110 & 10110001 \\
\hline 48 & 00110000 & 00000111 & 00110111 & 11001000 \\
\hline 81 & 01010001 & 00000101 & 01010100 & 10101000 \\
\hline 58 & 00111010 & 00000111 & 00111101 & 11000010 \\
\hline 53 & 00110101 & 00000101 & 00110000 & 11001111 \\
\hline 66 & 01000010 & 00000111 & 01000101 & 10111010 \\
\hline 37 & 00100101 & 00000101 & 00100000 & 11011111 \\
\hline 46 & 00100100 & 00000111 & 00100011 & 11011100 \\
\hline 90 & 01011010 & 00000101 & 01011111 & 10100000 \\
\hline 58 & 00111010 & 00000111 & 00111101 & 11000010 \\
\hline 47 & 00101111 & 00000101 & 00101010 & 11010101 \\
\hline 41 & 00101001 & 00000111 & 00101110 & 11010001 \\
\hline 85 & 01010101 & 00000101 & 01010000 & 10101111 \\
\hline 58 & 00111010 & 00000111 & 00111101 & 11000010 \\
\hline 75 & 01001011 & 00000101 & 01001110 & 10110001 \\
\hline 53 & 00110101 & 00000111 & 00110010 & 11001101 \\
\hline 92 & 01011100 & 00000101 & 01011011 & 10100100 \\
\hline 58 & 00111010 & 00000111 & 00111101 & 11000010 \\
\hline 57 & 00111001 & 00000101 & 00111100 & 11000011 \\
\hline 85 & 01010101 & 00000111 & 01010010 & 10101101 \\
\hline 48 & 00110000 & 00000101 & 00110101 & 11001010 \\
\hline 58 & 00111010 & 00000111 & 00111101 & 11000010 \\
\hline 61 & 00111101 & 00000101 & 00111000 & 11000111 \\
\hline 85 & 01010101 & 00000111 & 01010010 & 10101101 \\
\hline 80 & 01010000 & 00000101 & 01010101 & 10101010 \\
\hline 58 & 00111010 & 00000111 & 00111101 & 11000010 \\
\hline 81 & 01010001 & 00000101 & 01010100 & 10101011 \\
\hline 52 & 00110100 & 00000111 & 00110011 & 11001100 \\
\hline 71 & 01000111 & 00000101 & 01000010 & 10111101 \\
\hline 59 & 00111011 & 00000111 & 00111100 & 11000011 \\
\hline
\end{tabular}

Table 3. AES encryption

\begin{tabular}{cccc}
\hline Hexadecimal & AES S-Box (W1) & Binary & Cipher text \\
\hline AD & 95 & 10010101 & 149 \\
B6 & $4 \mathrm{e}$ & 01001110 & 78 \\
84 & $5 \mathrm{f}$ & 01011111 & 95 \\
A7 & $5 \mathrm{c}$ & 01011100 & 92 \\
C2 & 25 & 00100101 & 37 \\
81 & $0 \mathrm{c}$ & 00001100 & 12 \\
B4 & $8 \mathrm{~d}$ & 10001101 & 141 \\
B6 & $4 \mathrm{e}$ & 01001110 & 78 \\
A8 & $\mathrm{c} 2$ & 11000010 & 194 \\
A7 & $5 \mathrm{c}$ & 01011100 & 92 \\
C2 & $2 \mathrm{e}$ & 00101110 & 46 \\
DE & $1 \mathrm{~d}$ & 00011101 & 29 \\
DC & 86 & 10000110 & 134 \\
D0 & 70 & 01110000 & 112 \\
CC & $4 \mathrm{~b}$ & 01001011 & 75 \\
CF & $8 \mathrm{a}$ & 10001010 & 138 \\
C2 & 25 & 00100101 & 37 \\
\hline
\end{tabular}

KED-AES algorithm: combined key encryption decryption and advance ... (Edwin R. Arboleda) 
Table 3. AES encryption (continued)

\begin{tabular}{|c|c|c|c|}
\hline Hexadecimal & AES S-Box (W1) & Binary & Cipher text \\
\hline $\mathrm{CF}$ & $8 \mathrm{a}$ & 10001010 & 138 \\
\hline D1 & $3 e$ & 00111110 & 62 \\
\hline B1 & $\mathrm{c} 8$ & 11001000 & 200 \\
\hline $\mathrm{C} 8$ & e8 & 11101000 & 232 \\
\hline A8 & $\mathrm{c} 2$ & 11000010 & 194 \\
\hline $\mathrm{C} 2$ & 25 & 00100101 & 37 \\
\hline $\mathrm{CF}$ & $8 a$ & 10001010 & 138 \\
\hline BA & $\mathrm{f} 4$ & 11110100 & 244 \\
\hline DF & $9 \mathrm{e}$ & 10011110 & 158 \\
\hline $\mathrm{DC}$ & 86 & 10000110 & 134 \\
\hline A0 & $\mathrm{e} 0$ & 11100000 & 224 \\
\hline $\mathrm{C} 2$ & 25 & 00100101 & 37 \\
\hline D5 & 03 & 00000011 & 3 \\
\hline D1 & $3 e$ & 00111110 & 62 \\
\hline $\mathrm{AF}$ & 79 & 01111001 & 121 \\
\hline $\mathrm{C} 2$ & 25 & 00100101 & 37 \\
\hline B1 & c8 & 11001000 & 200 \\
\hline $\mathrm{CD}$ & $\mathrm{db}$ & 11011011 & 219 \\
\hline A4 & 49 & 01001001 & 73 \\
\hline $\mathrm{C} 2$ & 25 & 00100101 & 37 \\
\hline $\mathrm{C} 3$ & $2 \mathrm{e}$ & 00101110 & 46 \\
\hline $\mathrm{AD}$ & 95 & 10010101 & 149 \\
\hline CA & 74 & 01110100 & 116 \\
\hline $\mathrm{C} 2$ & 25 & 00100101 & 37 \\
\hline $\mathrm{C} 7$ & c6 & 11000110 & 198 \\
\hline $\mathrm{AD}$ & 95 & 10010101 & 149 \\
\hline AA & ac & 10101100 & 172 \\
\hline $\mathrm{C} 2$ & 25 & 00100101 & 37 \\
\hline $\mathrm{AB}$ & 62 & 01100010 & 98 \\
\hline $\mathrm{CC}$ & $4 b$ & 01001011 & 75 \\
\hline $\mathrm{BD}$ & $7 \mathrm{a}$ & 01111010 & 122 \\
\hline $\mathrm{C} 3$ & $2 \mathrm{e}$ & 00101110 & 46 \\
\hline
\end{tabular}

Decryption:

Table 4. AES decryption

\begin{tabular}{|c|c|c|c|c|}
\hline Decimal & Binary & Hexadecimal & Inverse S-Box (W2) & Binary \\
\hline 149 & 10010101 & 95 & $\mathrm{AD}$ & 10101101 \\
\hline 78 & 01001110 & $4 \mathrm{e}$ & B6 & 10110110 \\
\hline 95 & 01011111 & $5 f$ & 84 & 10000100 \\
\hline 92 & 01011100 & $5 \mathrm{c}$ & A7 & 10100111 \\
\hline 37 & 00100101 & 25 & $\mathrm{C} 2$ & 11000010 \\
\hline 12 & 00001100 & $0 \mathrm{c}$ & 81 & 10000001 \\
\hline 141 & 10001101 & $8 d$ & B4 & 10110100 \\
\hline 78 & 01001110 & $4 e$ & B6 & 10110110 \\
\hline 194 & 11000010 & $\mathrm{c} 2$ & A8 & 10101000 \\
\hline 92 & 01011100 & $5 \mathrm{c}$ & A7 & 10100111 \\
\hline 46 & 00101110 & $2 \mathrm{e}$ & $\mathrm{C} 2$ & 11000010 \\
\hline 29 & 00011101 & $1 d$ & $\mathrm{DE}$ & 11011110 \\
\hline 134 & 10000110 & 86 & DC & 11011100 \\
\hline 112 & 01110000 & 70 & D0 & 11010000 \\
\hline 75 & 01001011 & $4 \mathrm{~b}$ & $\mathrm{CC}$ & 11001100 \\
\hline 138 & 10001010 & $8 \mathrm{a}$ & $\mathrm{CF}$ & 11001111 \\
\hline 37 & 00100101 & 25 & $\mathrm{C} 2$ & 11000010 \\
\hline 138 & 10001010 & $8 \mathrm{a}$ & $\mathrm{CF}$ & 11001111 \\
\hline 62 & 00111110 & $3 e$ & D1 & 11010001 \\
\hline 200 & 11001000 & c8 & B1 & 10110001 \\
\hline 232 & 11101000 & e8 & $\mathrm{C} 8$ & 11001000 \\
\hline 194 & 11000010 & $\mathrm{c} 2$ & A 8 & 10101000 \\
\hline 37 & 00100101 & 25 & $\mathrm{C} 2$ & 11000010 \\
\hline 138 & 10001010 & $8 \mathrm{a}$ & $\mathrm{CF}$ & 11001111 \\
\hline 244 & 11110100 & $\mathrm{f} 4$ & BA & 10111010 \\
\hline 158 & 10011110 & $9 \mathrm{e}$ & DF & 11011111 \\
\hline 134 & 10000110 & 86 & DC & 11011100 \\
\hline 224 & 11100000 & $\mathrm{e} 0$ & A0 & 10100000 \\
\hline 37 & 00100101 & 25 & $\mathrm{C} 2$ & 11000010 \\
\hline 3 & 00000011 & 03 & D5 & 11010101 \\
\hline 62 & 00111110 & $3 \mathrm{e}$ & D1 & 11010001 \\
\hline
\end{tabular}

Int. J. of Adv. in Appl. Sci. Vol. 8, No. 1, March 2019: 44 - 53 
Int. J. of Adv. in Appl. Sci.

ISSN: $2252-8814$

Table 4. AES decryption (continued)

\begin{tabular}{ccccc}
\hline Decimal & Binary & Hexadecimal & Inverse S-Box (W2) & Binary \\
\hline 121 & 01111001 & 79 & AF & 10101111 \\
37 & 00100101 & 25 & $\mathrm{C} 2$ & 11000010 \\
200 & 11001000 & $\mathrm{c} 8$ & $\mathrm{~B} 1$ & 10110001 \\
219 & 11011011 & $\mathrm{db}$ & $\mathrm{CD}$ & 11001101 \\
73 & 01001001 & 49 & $\mathrm{~A} 4$ & 10100100 \\
37 & 00100101 & 25 & $\mathrm{C} 2$ & 11000010 \\
46 & 00101110 & $2 \mathrm{e}$ & $\mathrm{C} 3$ & 11000011 \\
149 & 10010101 & 95 & $\mathrm{AD}$ & 10101101 \\
116 & 01110100 & 74 & $\mathrm{CA}$ & 11001010 \\
37 & 00100101 & 25 & $\mathrm{C} 2$ & 11000010 \\
198 & 11000110 & $\mathrm{c} 6$ & $\mathrm{C} 7$ & 11000111 \\
149 & 10010101 & 95 & $\mathrm{AD}$ & 10101101 \\
172 & 10101100 & $\mathrm{ac}$ & $\mathrm{AA}$ & 10101010 \\
37 & 00100101 & 25 & $\mathrm{C} 2$ & 11000010 \\
98 & 01100010 & 62 & $\mathrm{AB}$ & 10101011 \\
75 & 01001011 & $4 \mathrm{~b}$ & $\mathrm{CC}$ & 11001100 \\
122 & 01111010 & $7 \mathrm{a}$ & $\mathrm{BD}$ & 10111101 \\
46 & 00101110 & $2 \mathrm{e}$ & $\mathrm{C} 3$ & 11000011 \\
\hline
\end{tabular}

Table 5. CHAOS decryption

\begin{tabular}{|c|c|c|c|c|}
\hline 1's Complement (S) & $\mathrm{J} 1 \& \mathrm{~J} 2$ & S XOR J1/J2 (S1) & Decimal & ASCII Code \\
\hline 01010010 & 00000111 & 01010101 & 85 & $\mathrm{U}$ \\
\hline 01001001 & 00000101 & 01001100 & 76 & $\mathrm{~L}$ \\
\hline 01111011 & 00000111 & 01111100 & 124 & | \\
\hline 01011000 & 00000101 & 01011101 & 93 & ] \\
\hline 00111101 & 00000111 & 00111010 & 58 & : \\
\hline 01111110 & 00000101 & 01111010 & 123 & \{ \\
\hline 01001011 & 00000111 & 01001100 & 76 & $\mathrm{~L}$ \\
\hline 01001001 & 00000101 & 01001100 & 76 & $\mathrm{~L}$ \\
\hline 01010111 & 00000111 & 01010000 & 80 & $\mathrm{P}$ \\
\hline 01011000 & 00000101 & 01011101 & 93 & ] \\
\hline 00111101 & 00000111 & 00111010 & 58 & : \\
\hline 00100001 & 00000101 & 00100100 & 36 & $\$$ \\
\hline 00100011 & 00000111 & 00100100 & 46 & . \\
\hline 00101111 & 00000101 & 00101010 & 42 & $*$ \\
\hline 00110011 & 00000111 & 00110100 & 52 & 4 \\
\hline 00110000 & 00000101 & 00110101 & 53 & 5 \\
\hline 00111101 & 00000111 & 00111010 & 58 & : \\
\hline 00110000 & 00000101 & 00110101 & 53 & 5 \\
\hline 00101110 & 00000111 & 00101001 & 41 & ) \\
\hline 01001110 & 00000101 & 01001011 & 75 & $\mathrm{~K}$ \\
\hline 00110111 & 00000111 & 00110000 & 48 & 0 \\
\hline 01010100 & 00000101 & 01010001 & 81 & Q \\
\hline 00111101 & 00000111 & 00111010 & 58 & : \\
\hline 00110000 & 00000101 & 00110101 & 53 & 5 \\
\hline 01000101 & 00000111 & 01000010 & 66 & V \\
\hline 00100000 & 00000101 & 00100101 & 37 & $\%$ \\
\hline 00100011 & 00000111 & 00100100 & 46 & . \\
\hline 01011111 & 00000101 & 01011010 & 90 & Z \\
\hline 00111101 & 00000111 & 00111010 & 58 & : \\
\hline 00101010 & 00000101 & 00101111 & 47 & / \\
\hline 00101110 & 00000111 & 00101001 & 41 & ( \\
\hline 01010000 & 00000101 & 01010101 & 85 & U \\
\hline 00111101 & 00000111 & 00111010 & 58 & : \\
\hline 01001110 & 00000101 & 01001011 & 75 & $\mathrm{~K}$ \\
\hline 00110010 & 00000111 & 00110101 & 53 & 5 \\
\hline 01011011 & 00000101 & 01011100 & 92 & 1 \\
\hline 00111101 & 00000111 & 00111010 & 58 & : \\
\hline 00111100 & 00000101 & 00111001 & 57 & 9 \\
\hline 01010010 & 00000111 & 01010101 & 85 & $\mathrm{U}$ \\
\hline 00110101 & 00000101 & 00110000 & 48 & 0 \\
\hline 00111101 & 00000111 & 00111010 & 58 & : \\
\hline 00111000 & 00000101 & 00111101 & 61 & $=$ \\
\hline 01010010 & 00000111 & 01010101 & 85 & $\mathrm{U}$ \\
\hline 01010101 & 00000101 & 01010000 & 80 & $\mathrm{P}$ \\
\hline 00111101 & 00000111 & 00111010 & 58 & : \\
\hline 01010100 & 00000101 & 01010001 & 81 & Q \\
\hline 00110011 & 00000111 & 00110100 & 52 & 4 \\
\hline 01000010 & 00000101 & 01000111 & 71 & G \\
\hline 00111100 & 00000111 & 00111011 & 59 & ; \\
\hline
\end{tabular}


Table 6. KED decryption

\begin{tabular}{|c|c|c|c|c|}
\hline Integer Value (V2) & V2-K2 (P1) & $\mathrm{P} 1{ }^{*} \mathrm{n} 1(\mathrm{P} 2)$ & P2 mod69 & $\begin{array}{l}\text { Plaintext (PT) } \\
\end{array}$ \\
\hline 21 & 15 & 210 & 3 & $\mathrm{C}$ \\
\hline 12 & 6 & 84 & 15 & $\mathrm{O}$ \\
\hline 67 & 61 & 854 & 26 & Z \\
\hline 62 & 56 & 784 & 25 & $\mathrm{Y}$ \\
\hline 53 & 47 & 658 & 37 & SPACE \\
\hline 66 & 60 & 840 & 12 & $\mathrm{~L}$ \\
\hline 12 & 6 & 84 & 15 & $\mathrm{O}$ \\
\hline 12 & 6 & 84 & 15 & $\mathrm{O}$ \\
\hline 16 & 10 & 140 & 2 & B \\
\hline 62 & 56 & 784 & 25 & $\mathrm{Y}$ \\
\hline 53 & 47 & 658 & 37 & SPACE \\
\hline 41 & 35 & 490 & 7 & G \\
\hline 51 & 45 & 630 & 9 & I \\
\hline 47 & 41 & 574 & 22 & V \\
\hline 31 & 25 & 350 & 5 & E \\
\hline 32 & 26 & 364 & 19 & $\mathrm{~S}$ \\
\hline 53 & 47 & 658 & 37 & SPACE \\
\hline 32 & 26 & 364 & 19 & $\mathrm{~S}$ \\
\hline 46 & 40 & 560 & 8 & $\mathrm{H}$ \\
\hline 11 & 5 & 70 & 1 & A \\
\hline 27 & 21 & 294 & 18 & $\mathrm{R}$ \\
\hline 17 & 11 & 154 & 16 & $\mathrm{P}$ \\
\hline 53 & 47 & 658 & 37 & SPACE \\
\hline 32 & 26 & 364 & 19 & $\mathrm{~S}$ \\
\hline 22 & 16 & 224 & 17 & Q \\
\hline 42 & 36 & 504 & 21 & $\mathrm{U}$ \\
\hline 51 & 45 & 630 & 9 & I \\
\hline 26 & 20 & 280 & 4 & D \\
\hline 53 & 47 & 658 & 37 & SPACE \\
\hline 52 & 46 & 644 & 23 & W \\
\hline 46 & 40 & 560 & 8 & $\mathrm{H}$ \\
\hline 12 & 6 & 84 & 15 & $\mathrm{O}$ \\
\hline 53 & 47 & 658 & 37 & SPACE \\
\hline 11 & 5 & 70 & 1 & A \\
\hline 32 & 26 & 364 & 19 & $\mathrm{~S}$ \\
\hline 61 & 55 & 770 & 11 & K \\
\hline 53 & 47 & 658 & 37 & SPACE \\
\hline 36 & 30 & 420 & 6 & $\mathrm{~F}$ \\
\hline 12 & 6 & 84 & 15 & $\mathrm{O}$ \\
\hline 27 & 21 & 294 & 18 & $\mathrm{R}$ \\
\hline 53 & 47 & 658 & 37 & SPACE \\
\hline 56 & 50 & 700 & 10 & $\mathrm{~J}$ \\
\hline 12 & 6 & 84 & 15 & $\mathrm{O}$ \\
\hline 16 & 10 & 140 & 2 & B \\
\hline 53 & 47 & 658 & 37 & SPACE \\
\hline 17 & 11 & 154 & 16 & P \\
\hline 31 & 25 & 350 & 5 & E \\
\hline 7 & 1 & 14 & 14 & $\mathrm{~N}$ \\
\hline 54 & 48 & 672 & 51 & \\
\hline
\end{tabular}

\section{CONCLUSION}

The proposed cryptosystem was able to combine the KED using modulo 69, CHAOS-based and AES cryptosystem. It is the enhanced system of KED by adding the chaotic-based cryptosystem and the AES S-box and inverse S-box in both encryption and decryption. The proposed algorithm is then implemented by using a message that comprise the English alphabet. The key is generated using the proposed method. Then the message is encrypted and decrypted successfully.

\section{REFERENCE}

[1] S. Hebert, "A Brief History of Cryptography”, an article [Online]. Available: http://cybercrimes.net/aindex.html.

[2] A. Sanada, Y. Nogami, K. Iokibe, and A. Khandaker, "Security Analysis of Raspberry Pi Against Side-Channel Attack with RSA Cryptography," pp. 287-288, 2017.

[3] Trevisan, L., "Cryptography. Lecture Notes from CS276," Spring 2009, vol. 154, 2009.

[4] L. K. Galla, V. S. Koganti, and N. Nuthalapati, "Implementation of RSA," in 2016 International Conference on Control Instrumentation Communication and Computational Technologies, ICCICCT 2016, pp. 81-87, 2017.

Int. J. of Adv. in Appl. Sci. Vol. 8, No. 1, March 2019: 44 - 53 
[5] D. Llamocca, D. Ph, D. Debnath, D. Ph, L. Rong, and D. Ph, "Can Crypto Chip To Secure Data Transmitted Through Can Fd Bus by Using Aes-128 \& Sha-1 With A Symmetric Key by Tri P. Doan," A dissertation submitted in partial fulfillment of the requirement for the degree of Doctor of Philosophy Rochester, Michigan Doct, 2017.

[6] Goldwasser, S., and Bellare, M., "Lecture Notes on Cryptography," 2008.

[7] S. Oukili and S. Bri, "High throughput FPGA Implementation of Data Encryption Standard with time variable subkeys," Int. J. Electr. Comput. Eng., vol. 6, no. 1, p. 298, 2016.

[8] S. Dey, J. Nath, and A. Nath, "An Integrated Symmetric Key Cryptographic Method - Amalgamation of TTJSA Algorithm, Advanced Caesar Cipher Algorithm, Bit Rotation and Reversal Method: SJA Algorithm," Int. J. Mod. Educ. Comput. Sci., vol. 4, no. 5, pp. 1-9, 2012.

[9] P. K. Panda, "A Hybrid Security Algorithm for RSA Cryptosystem,” 2017.

[10] Caesar, G. J. and Kennedy, J.F. (n.d.)., "Cryptography. Security Engineering: A Guide to Building Dependable Distributed Systems," vol. 42, 2008.

[11] D. John and L. Martin, "Binary rsa encryption algorithm,” pp. 178-181, 2016.

[12] L. Kocarev, J.M. Amigo, and J. Szczepanski, "The Chaos-based Cryptography: an overview", IEEE Circuits and Systems Magazine, vol. 1(3), pp. 6-21, 2001.

[13] Y. Chen, K. Li, X. Fei, Z. Quan, and K. Li, "Implementation and Optimization of AES Algorithm on the Sunway Taihu Light," 2016.

[14] K. Garson, Policy-Based Encryption and its Application in a Hospital System by. 2009.

[15] Q. Zhang and A. Qunding, "Digital image encryption based on Advanced Encryption Standard(AES) algorithm," 5th Int. Conf. Instrum. Meas. Comput. Commun. Control. IMCCC 2015, pp. 1218-1221, 2015.

[16] J.-F. W. J.-F. Wang, S.-W. C. S.-W. Chang, and P.-C. L. P.-C. Lin, "A novel round function architecture for AES encryption/decryption utilizing look-up table," IEEE 37th Annu. 2003 Int. Carnahan Conf. onSecurity Technol. 2003 Proc., pp. 132-136, 2003.

[17] P. Katkade and G. M. Phade, "Application of AES algorithm for data security in serial communication," Proc. Int. Conf. Inven. Comput. Technol. ICICT 2016, vol. 2016, 2016.

[18] O. A. Dawood, A. M. S. Rahma, and A. M. J. Abdul Hossen, "New Symmetric Cipher Fast Algorithm of Revertible Operations' Queen (FAROQ) Cipher," Int. J. Comput. Netw. Inf. Secur., vol. 9, no. 4, pp. 29-36, 2017.

[19] J, Daemen and V. Rijmen, “AES Proposal: Rijndael, AES Algorithm Submission,” September 3, 1999.

[20] J. Daemen and V. Rijmen, "The block cipher Rijndael, Smart Card research and Applications, LNCS 1820," Springer-Verlag, pp. 288-296.

[21] A. K. Sharma and H. Sharma, "New Approach to Des with Enhanced Key Management and Encryption/Decryption System," vol. 32, pp. 1-31, 2012.

[22] J. Warjri, KED- “A Symmetric Key Algorithm for Secured Information Exchange Using Modulo 69, ” I.J Computer Network and Information Security, vol. 10, pp 37-43, 2013.

[23] J. L. Lai, K. H. Liao, Y. Te Lai, and R. J. Chen, "Design CAROM module used in AES structure for Sub-Byte and Inv-Sub-Byte transformation," Proc. - 2013 Int. Symp. Biometrics Secur. Technol. ISBAST 2013, no. 1, pp. 198-202, 2013. 